\title{
Angiogenesis in the Mouse Lung
}

\author{
Wayne Mitzner, Won Lee, \\ Dimitrios Georgakopoulos, and Elizabeth Wagner \\ From the Departments of Medicine and Environmental Health \\ Sciences, The Johns Hopkins University, Baltimore, Maryland
}

\begin{abstract}
When pulmonary arterial blood flow is obstructed in all mammals studied, there is a compensatory growth of the bronchial vasculature. This angiogenesis normally occurs through a proliferation of the systemic circulation to the intraparenchymal airways. It is an important pathophysiological process, not only in pulmonary vascular disease, but also in lung cancer, because the blood flow that supplies primary lung tumors arises from the systemic circulation. In the mouse, however, the systemic blood vessels that supply the trachea and mainstem bronchi do not penetrate into the intraparenchymal airways, as they do in all other larger species. In this study, we attempted to generate a new functional bronchial circulation in the mouse by permanently obstructing $40 \%$ of the pulmonary circulation. We quantified the systemic blood flow to the lung with fluorescent microspheres for 3 months after left pulmonary artery ligation. Results demonstrated that a substantial systemic blood flow to the lung that can eventually supply up to $15 \%$ of the normal pulmonary flow can be generated beginning 5-6 days after ligation. These new angiogenic vessels do not arise from the extraparenchymal bronchial circulation. Rather they enter the lung directly via a totally new vasculature that develops between the visceral and parietal pleuras, supplied by several intercostal arteries. This unique model of angiogenesis occurs in the absence of any hypoxic stimulus and mimics the vascular source of many lung tumors.
\end{abstract} (Am J Patbol 2000, 157:93-101)

When pulmonary arterial blood flow is obstructed in all mammals studied, there is a compensatory growth of the bronchial circulation. ${ }^{1-3}$ This increased systemic blood flow to the lung can increase to as much as 30\% of the original pulmonary blood flow. ${ }^{3}$ There are a host of growth factors, hormones, and chemokines that regulate angiogenesis,, 4 but our understanding of the physiological integration involved remains highly speculative in both systemic tissues and the lung. Although there are systemic blood vessels that supply the trachea down to the carina and mainstem bronchi, the mouse lung does not have a normal systemic circulation to the intraparenchymal airways. ${ }^{6}$ We questioned whether a new func- tional bronchial circulation might be generated in this species if one pulmonary artery were obstructed.

In addition to its functional consequences, this question also has relevance to the vascular supply of lung tumors, because the blood flow that supplies lung tumors can arise from either the pulmonary or systemic circulation. The conventional wisdom is that angiogenesis into lung tumors occurs primarily through the bronchial circulation, ${ }^{7,8}$ but because of anastomoses between the bronchial and pulmonary circulations, embolization of the bronchial circulation is not effective in killing lung tumors. The pulmonary vasculature can thus function as a maintenance vasculature for lung tumors, but with little capacity for angiogenesis; the systemic vasculature provides the primary source for new vessel growth. Atthough this schema may not always occur in metastatic lung tumors, ${ }^{9}$ there is general agreement implicating the systemic circulation in the lung as the initiator of the blood supply to primary lung tumors. ${ }^{7-9}$ How this process would operate in the mouse, however, is unclear, because in this species there is no systemic circulation to the airways or parenchyma. ${ }^{6}$ Thus for the conventional model of lung tumor vascularization to be applicable in mice, there must be some other source of systemic blood flow that can initiate the angiogenesis. There is little discussion of this problem in the literature, but one potential source of this systemic blood supply to primary tumors is adhesions to the mediastinum or thoracic wall. ${ }^{10}$

In the present study, we describe a new experimental model in the mouse that completely isolates this angiogenic process from tumor development. In this model a new circulation was generated by obstructing one pulmonary artery. Unlike in other species, this new circulation does not arise from the existing systemic vessels in the extraparenchymal airways. Rather the vessels enter the lung directly via a totally new vasculature penetrating the visceral pleural surface. The model thus mimics the vascular source of many lung tumors and allows independent investigation of those factors that can promote or inhibit angiogenesis.

Supported by National Institutes of Health grants NHLBI 10342 and NIEHS 03819 .

Accepted for publication March 3, 2000

Address reprint requests to Dr. Wayne Mitzner, Division of Physiology, The Johns Hopkins School of Hygiene and Public Health, 615 N. Wolfe Street, Baltimore, MD 21205. E-mail: wmitzner@jhsph.edu. 


\section{Materials and Methods}

\section{Occlusion of Left Pulmonary Artery}

Male C57BL/6J ( $n=30$; Jackson Laboratories, Bar Harbor, ME) mice 6 weeks old were used in this study. All animals were sedated with a metofane-soaked sponge in a sealed container and then deeply anesthetized with an intraperitoneal injection of ketamine (135 mg/kg) + acepromazine $(1.5 \mathrm{mg} / \mathrm{kg})$. They were intubated and mechanically ventilated with $100 \%$ oxygen by a ventilation procedure previously described, ${ }^{11}$ with a tidal volume of $0.2 \mathrm{ml}$ at a rate of 120 breaths/minute. After topical lidocaine, a left thoracotomy was performed in the third intercostal space. The left main pulmonary artery was identified, a 6-O silk suture was placed around it, and then the vessel was ligated. A ligature was place around the third and fourth ribs, and cyanoacrylate adhesive (Future Glue; Pacer Technologies, Rancho Cucamonga, CA) was applied to the cut surface. The thorax was then sealed by tightening the ligature. The animals were extubated and allowed to recover on a heating pad. A subcutaneous injection of $0.2 \mathrm{ml}$ of lactated Ringer's solution was given to supplement fluid volume. Animal protocols used in this study were reviewed and approved by the Johns Hopkins Institutions Animal Care and Use Committee and were conducted using national guidelines for the care and protection of animals.

\section{Assessment of Perfusion}

Vascular perfusion of the left lung was compared to that in the right lung for 12 weeks after left pulmonary artery ligation. Perfusion in individual animals was measured daily for the first week and then at weekly or longer intervals. The extent of perfusion was quantified using $15-\mu \mathrm{m}$ fluorescently labeled microspheres (Interactive Medical Technologies, Los Angeles, CA; ips1@imt-Itd.com). To measure blood flow, animals were anesthetized with $1000 \mathrm{mg} / \mathrm{kg}$ urethane and $5 \mathrm{mg} / \mathrm{kg}$ etomidate injected i.p. and mechanically ventilated after tracheostomy. After a small incision in the neck, a jugular vein was dissected free and directly cannulated with a 31-gauge needle connected to PE-10 tubing and a $1-\mathrm{ml}$ syringe. Forty thousand spheres $(0.2 \mathrm{ml})$ of one color were first injected into the jugular vein at a rate of $40 \mu \mathrm{l} /$ minute. When this was completed, the thorax was opened from the abdomen by cutting along the ventral attachments of the diaphragm. Approximately $50 \mu \mathrm{l}$ of a 50 $\mu \mathrm{g} / \mathrm{ml}$ solution of epinephrine was dripped into the peritoneal cavity to help maintain cardiac function. One hundred thousand spheres $(0.1 \mathrm{ml})$ of another color were injected into the left ventricle through the apex at a rate of $40 \mu \mathrm{l} /$ minute. Animals were then sacrificed with $50 \mu \mathrm{l}$ of a supersaturated $\mathrm{KCl}$ solution placed directly on the heart. The left and right lungs were excised, washed in fresh saline, and placed in labeled tubes for analysis of the number of spheres. At designated time points, we assessed the pulmonary and systemic perfusion to the left and right lungs in each of 20 mice in vivo. Lungs were digested with proteases to release the spheres, which were then purified through sucrose gradients. The number of spheres in each lung was
Table 1. Methacrylate Mixture Used to Cast Angiogenic Blood Vessels in Mice

\begin{tabular}{|c|c|c|c|}
\hline \multirow{2}{*}{$\frac{\text { Solution } 1}{\text { Batson's base }}$} & \multicolumn{3}{|c|}{ Solution 2} \\
\hline & $2.5 \mathrm{ml}$ & Batson's base & $2.5 \mathrm{ml}$ \\
\hline Methyl methacrylate & $3.7 \mathrm{ml}$ & Methyl methacrylate & $3.7 \mathrm{ml}$ \\
\hline Batson's catalyst & $5.0 \mathrm{ml}$ & Batson's promoter & $0.4 \mathrm{ml}$ \\
\hline Ethylene dimethacrylate & $0.6 \mathrm{ml}$ & & \\
\hline Acetone & $0.4 \mathrm{ml}$ & & \\
\hline \multicolumn{4}{|l|}{ Color } \\
\hline Blue and red & $0.1 \mathrm{~g}$ & & \\
\hline White & $0.2 \mathrm{~g}$ & & \\
\hline
\end{tabular}

This mixture yields $10 \mathrm{ml}$ total when the two solutions are mixed together. To get $1 \mathrm{ml}$ of cast, take $0.7 \mathrm{ml}$ of solution 1 and $0.3 \mathrm{ml}$ of solution 2. Stir well for 1 minute. Material will have a low viscosity for about 5 minutes.

assessed by flow cytometry with a calibrated reference sample (Interactive Medical Technologies). Because we did not obtain a reference sample from the aorta during infusion, this method of obtaining the total number of spheres per lung sample only provides fractional distributions, not absolute measurements of blood flow. From the injection into the jugular vein, we obtain the fraction of pulmonary artery flow to each lung. From the injection into the left ventricle, we obtain the fraction of cardiac output perfusing each lung.

\section{Lung Mechanics}

Immediately before we injected the microspheres into the jugular vein, the lungs were inflated to a static pressure of $25 \mathrm{cmH}_{2} \mathrm{O}$ for 4 seconds and then return to normal ventilation at functional residual capacity (FRC) for 1 minute. To assess whether the pulmonary artery ligation of $\sim 40 \%$ of the lung would lead to functional changes in lung mechanics, measurements of respiratory system compliance and resistance were then made, using the inspiratory occlusion method as previously described. ${ }^{11}$ Briefly, this method involves a 200-millisecond occlusion at the end of inspiration. The peak inspiratory pressure can then be partitioned into resistive and compliant components.

\section{Morphology}

In several animals 2-3 months after surgery, we injected methacrylate casting material retrograde into the descending aorta according to the following procedure. Mice were sacrificed with i.p. urethane $(2000 \mathrm{mg} / \mathrm{kg})$. The abdomen was opened, and the descending aorta was cannulated with PE-50 tubing filled with saline. The inferior vena cava was cut, and the systemic circulation was flushed with $1 \mathrm{ml}$ of saline. The saline syringe was then exchanged for one with $0.5 \mathrm{ml}$ of the methacrylate mixture (see Table 1), and this was injected manually at a rate sufficient to maintain an infusion pressure of $\sim 200$ $\mathrm{mmHg}$. The mouse lung was then inflated with $0.6 \mathrm{ml}$ formalin, and half an hour later the entire thorax was excised and placed in 10\% formalin. 

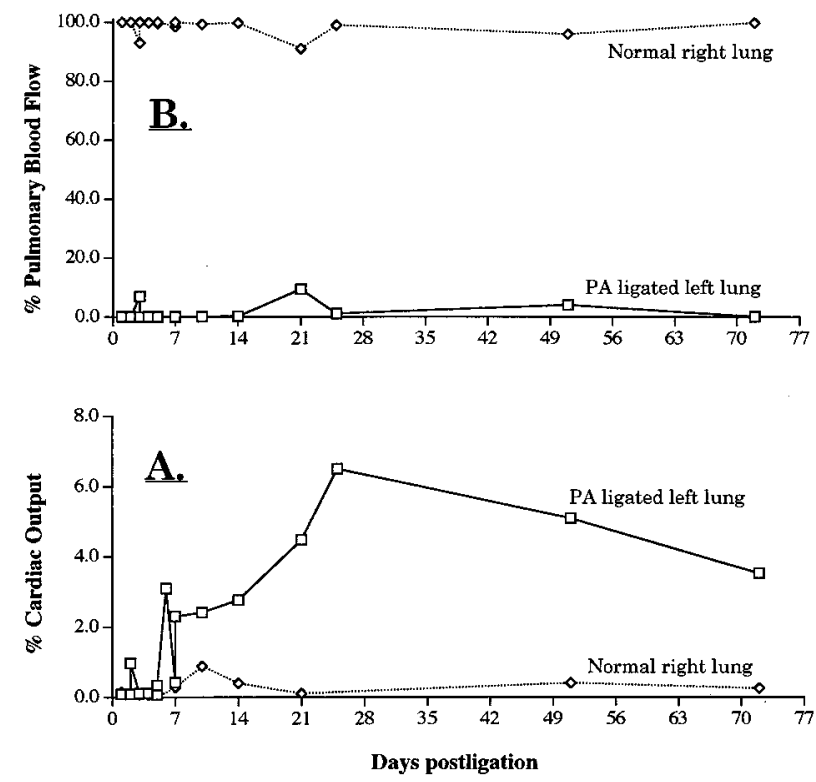

Figure 1. A: Systemic blood flow (as a percentage of cardiac output) to left and right lungs. B: Fraction of pulmonary blood flow to left and right lungs. Systemic blood flow to the ligated lung does not increase until 5-6 days after ligation, and it appears to stabilize in 2-3 weeks. Pulmonary blood flow to the left lung does not recover after ligation.

Two mice with tied pulmonary arteries were sacrificed with urethane as above, 2 days after ligation. Their lungs were filled with $0.6 \mathrm{ml}$ of $10 \%$ formalin and then excised and placed in formaldehyde for routine histology. Lungs were embedded in glycol methacrylate, sectioned at 3 $\mu \mathrm{m}$ and stained with $1 \%$ toluidine blue in $1 \%$ borax followed by $1 \%$ napthol yellow $S$ in $1 \%$ acetic acid.

\section{Results}

All animals survived ligation of the left PA. Usually within an hour of completion of surgery they were moving about in their cages. By the next day, their behavior was completely normal and could only be distinguished from control mice by the absence of fur at the incision site. In control mice, an average of $40 \%$ of the pulmonary blood flow went to the left lung. In the mice with the left PA tied, there was no significant pulmonary blood flow to the left lung. Systemic blood flow to the normal right lung was normally less than the background noise level of the measurement (200 spheres, or with the 100,000 that were injected, $0.2 \%$ of cardiac output). Tying the left PA did not increase the systemic blood flow to the right lung. Figure 1 shows the fractions of cardiac output and pulmonary blood flow going to the left and right lungs as a function of time after surgery. Each point in this figure (and in Figure 2) shows the measurement in individual mice. This figure shows an increase in measurable systemic blood flow to the left lung that began after day 5. This increased up to 2 weeks, where it seemed to reach a plateau at $\sim 5-6 \%$ of cardiac output.

Respiratory system compliances and resistances are shown in Figure 2. There is a slightly decreased compliance and increased resistance in the first week after
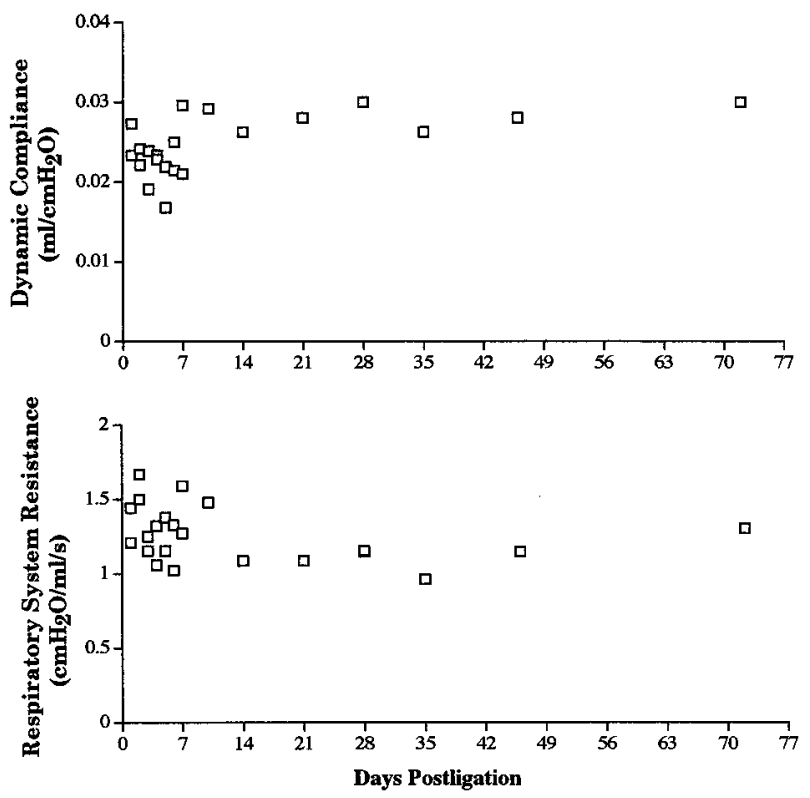

Figure 2. Respiratory system compliance (top) and resistance (bottom) as a function of days after ligation of the left pulmonary artery.

ligation. To compare the average compliances and resistances from mice during the first week after ligation to those measured in mice longer than 2 weeks after ligation we used an unpaired $t$-test. The average compliance ( \pm SEM) during the first week was $0.023 \pm 0.0008 \mathrm{ml} /$ $\mathrm{CmH}_{2} \mathrm{O}$, and that longer than 2 weeks after ligation was $0.028 \pm 0.0005(P=0.0013)$. The average resistances $( \pm$ SEM) over these same two time periods were $1.31 \pm$ 0.04 and $1.13 \pm 0.03(P=0.021)$, respectively.

On gross inspection, the left lung often appeared less pink than the right from day 1 to day 3 , but after day 4 the left lung color approached that of the right lung. There was no evidence of gross atelectasis or necrosis in the left lung at any time after surgery. In all mice, we observed angiogenic blood vessels arising from the thoracic wall and entering the lung through the visceral pleura. Figures 3-5 show examples from one of the animals injected with a red cast. Figure 3 shows the open thorax with the ventral surface of the left lung visible. There was always extensive new vascularization on the dorsal surface of the left lung near the third intercostal space. Enlarged intercostal arteries can be seen in the area near the new vascular attachments between the lung and chest wall. These arteries that now supply the lung show the characteristic tortuous course of angiogenic vascularization. Normal-sized intercostal arteries can be seen lower down the thoracic wall. Figure 4 shows the same thorax, with the left lung now partially retracted from the chest wall. The extensive network of anastomotic vessels between the visceral and parietal pleuras is now readily apparent. The figure shows an invagination of the upper part of the lung that is caused by the rigid cast vessels pulling the lung in as the lung is forcibly pulled away from the chest wall to illustrate the vessels. Figure 5 shows this same left lung now torn completely free of the anastomoses, pulled down, and turned over to show the dense vascularization on the dorsal 


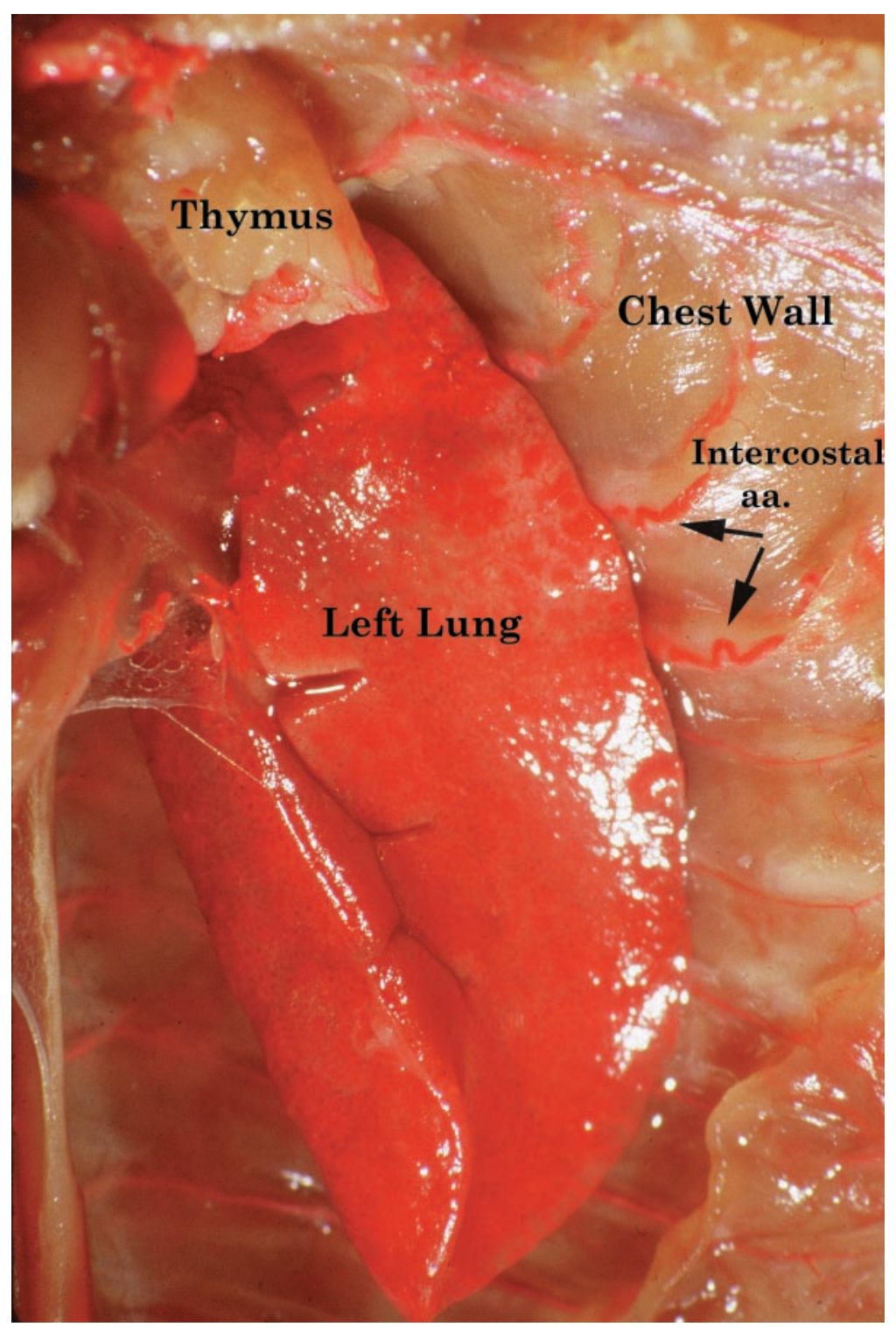

Figure 3. View of ventral surface of the left lung in the open thorax. Systemic vasculature was infused with a red-dyed methacrylate cast. Note tortuous dilated intercostal arteries adjacent to upper half of the lung. Intercostal arteries adjacent to lower half of the lung appear normal.

side and on the chest wall. In several sham-operated controls with no pulmonary artery ligation, none of these vascular changes were observed.

In addition to these new vessels that arose from the thoracic wall, we occasionally observed new vessels that arose from other systemic arteries and were attached directly to the apical or upper ventral surface of the lung. These vessels seemed to take a tortuous path through the thymus, before reaching the lung visceral surface. This type of new vessel to the ventral surface of the lung was rare, being observed in only four of the 30 mice examined.

Figure 6 shows the pleural surface of a normal mouse lung and that of the left lung 2 days after surgery in the region close to the third intercostal space. The normal mouse subpleural region is highly vascularized, with one or more blood vessels located at each junction of alveolar wall with the pleura. In the animal with ligated pulmonary artery, enlarged mesothelial cells, and subpleural blood vessels can be seen to have migrated outside the disrupted visceral pleura. The arrow in the figure shows one such example. There also appears to be some necrotic tissue and inflammatory cells in the thickened parenchymal regions near the pleural surface.

\section{Discussion}

When pulmonary arterial blood flow is obstructed in all mammals previously studied, there is a compensatory growth of the bronchial circulation ${ }^{1-3}$ that can increase to as much as $30 \%$ of the original pulmonary blood flow. ${ }^{3}$ This experiment had not been done in the mouse, but our present results clearly demonstrate that occlusion of the pulmonary circulation in mice also leads to new systemic vascularization of the lung. Unlike other species, how- 


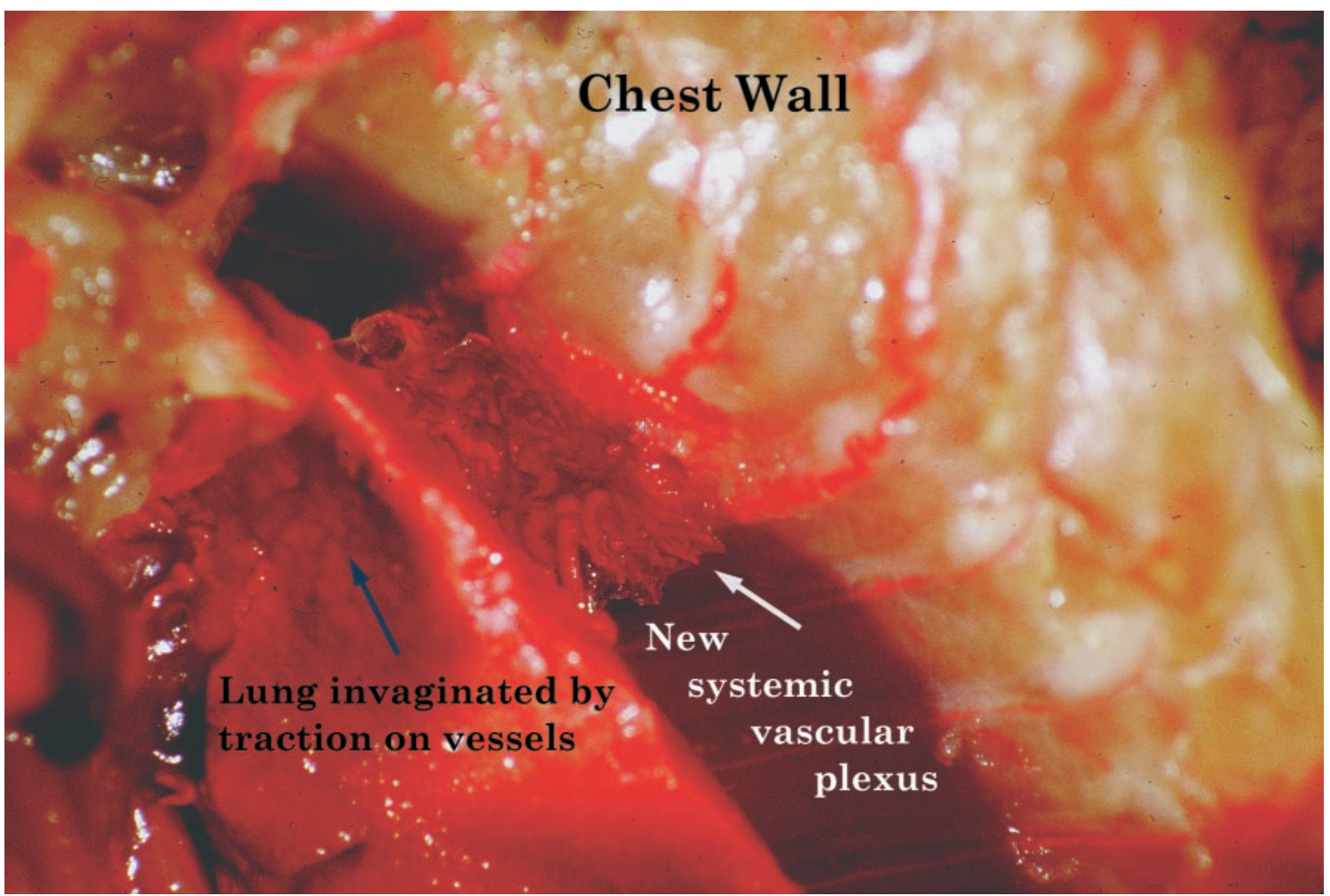

Figure 4. View of left lung partially retracted from the thoracic wall, showing the extensive new vascular plexus that connects the visceral and parietal pleuras.

ever, this new circulation does not arise from expansion of the tiny systemic blood vessels at the carina and mainstem bronchi. Rather, the new supply arises primarily from the parietal pleura fed from intercostal arteries. Measurable blood flow could be detected with 15- $\mu \mathrm{m}$ microspheres as early as the fifth day after PA ligation. The new systemic blood flow to the left lung appeared to stabilize in the range of $4-6 \%$ of cardiac output. Because the left lung normally receives $\sim 40 \%$ of pulmonary blood flow, this new systemic flow supplies $\sim 10-15 \%$ of the normal pulmonary flow. Apparently, this is more than sufficient to keep the lung tissues alive. In dogs, the increase in bronchial blood flow after left pulmonary artery ligation was measured by Michel et al. ${ }^{3}$ They found

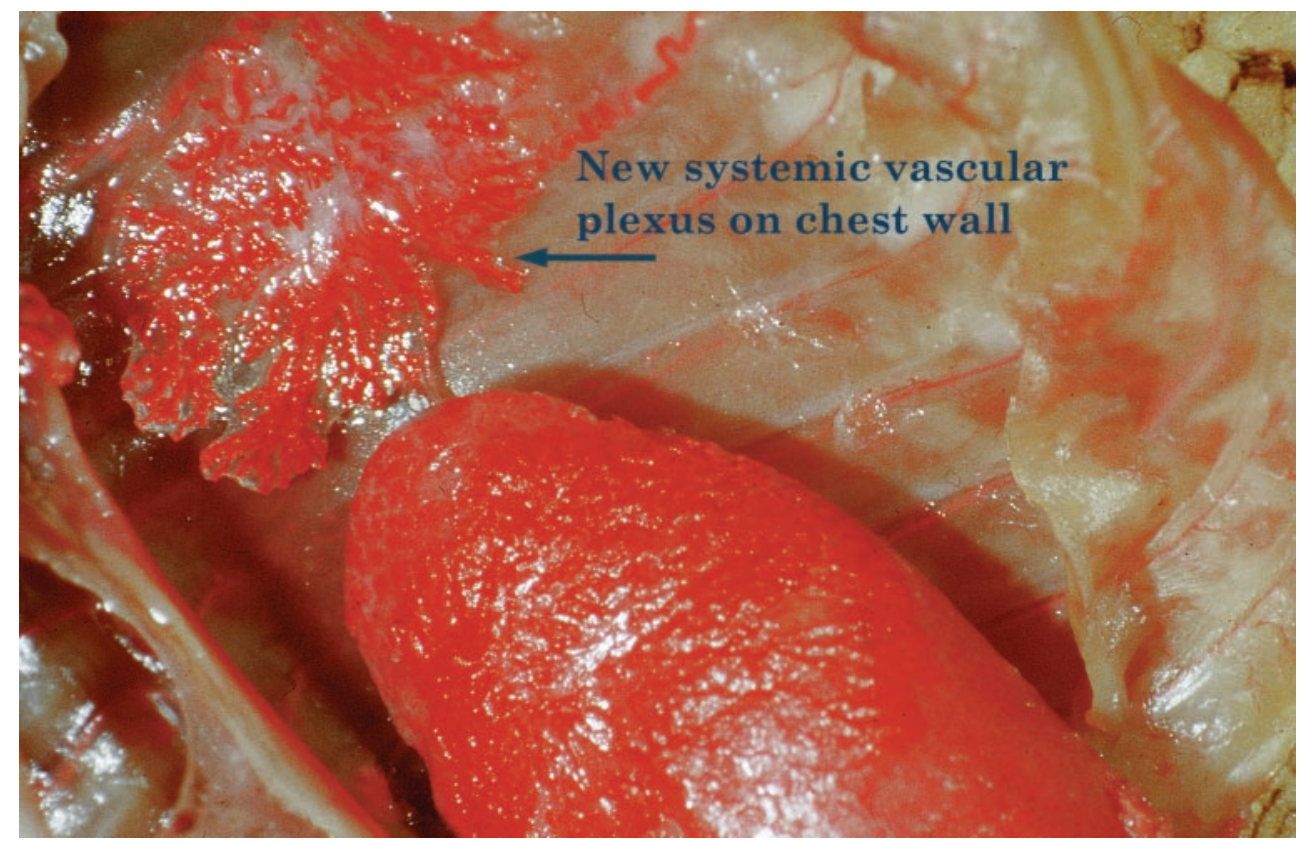

Figure 5. View of left thoracic wall after removal of the left lung. The left lung has been pulled down and turned over to show the dorsal surface that had been connected to the chest wall. 


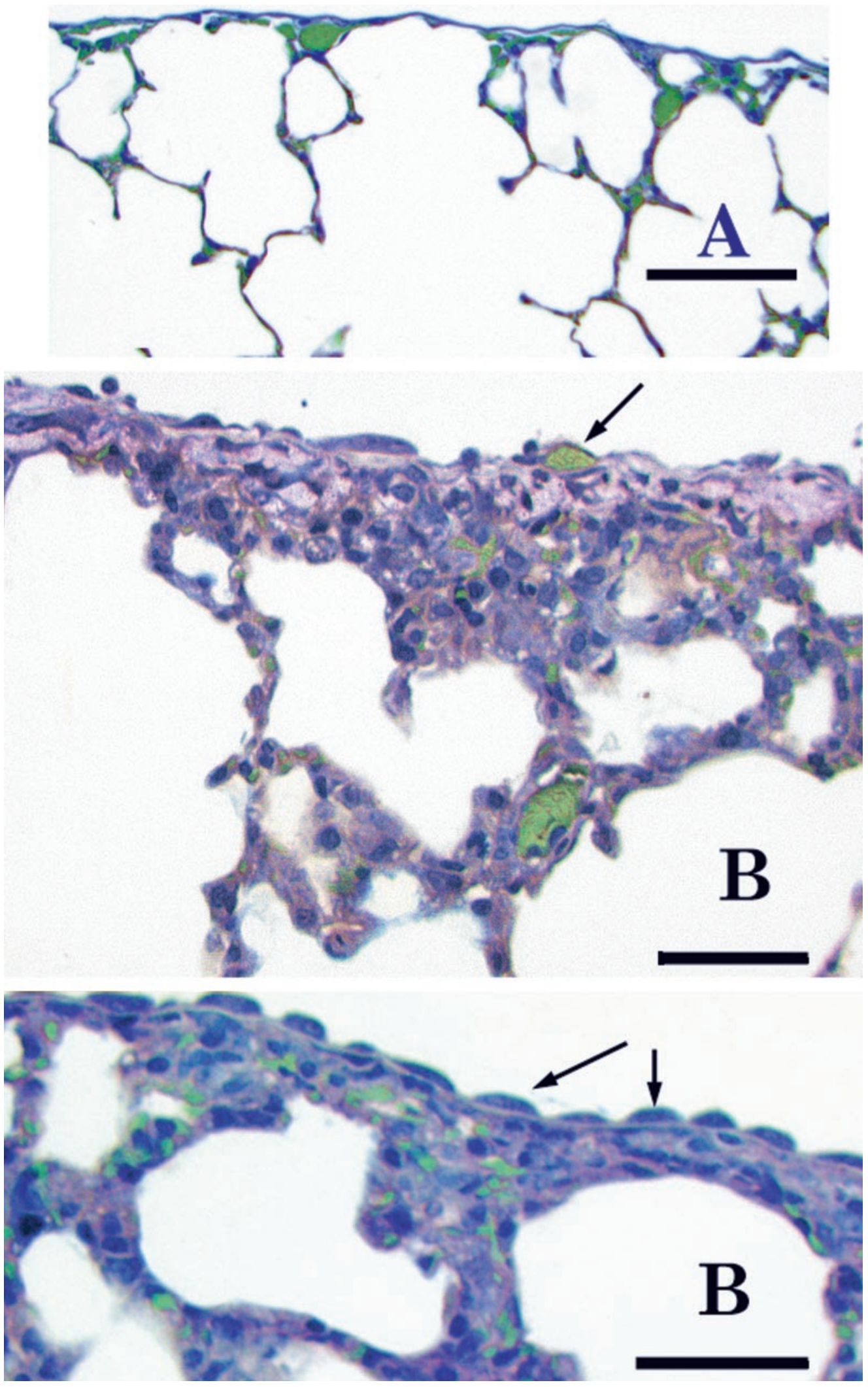

Figure 6. Histological sections of visceral pleura from the upper dorsal region of a normal left lung (A) and from the left lung of a mouse 2 days after ligation of the left pulmonary artery $(\mathbf{B})$. A: The normal mouse subpleural region is highly vascularized, with one or more blood vessels located at each junction of the alveolar wall with the pleura. Note that the red blood cells are stained green. Bar, $100 \mu \mathrm{m}$. B: The two sections are from different regions of the pleura in one animal that absorbed different amounts of toluidine blue stain. In the lower half, note enlarged mesothelial cells (arrows) on the pleural surface, and in the upper image there is a pleural blood vessel (arrow) located almost outside the disrupted visceral pleura. Bars, $50 \mu \mathrm{m}$. 
bronchial blood flows of $330 \mathrm{ml} /$ minute, 4 months after ligation. With a nominal $3 \mathrm{~L} /$ minute cardiac output and $40 \%$ normally going to the left lung, the increased bronchial blood flow after ligation represents almost 30\% of the normal pulmonary blood flow. This is about two to three times greater than the compensatory flow we found in mice. However, in sheep, the compensatory systemic flow was less than what we found in mice. The bronchial blood flow to the sheep left lung increased from 35 to 134 $\mathrm{ml} /$ minute 3 weeks after ligation of the left pulmonary artery. ${ }^{1}$ With a measured cardiac output of $\sim 5 \mathrm{~L} /$ minute, this postligation bronchial blood flow is thus slightly less than $3 \%$ of cardiac output. Thus the magnitude of the functional compensatory vascularization observed in the mouse is within the range of what has been found in larger animals.

We found slightly altered mechanics of the respiratory system during the first week after ligation, with the compliance lower and the resistance higher. Reasons for these temporary changes are not entirely clear. There may be some residual stiffness in the left chest wall resulting from the gluing together of two adjacent ribs that could contribute to decreased respiratory system stiffness. However, this would still not explain the increased respiratory resistance. Vascular engorgement is known to decrease the compliance of the lung, ${ }^{12}$ but because we have decreased the pulmonary vascular pressure after ligation, this mechanism does not seem to be of importance. Perhaps the absence of blood flow increases airway smooth muscle tone, but we have little evidence to support this speculation. Whatever the reason, when the blood flow increases to a new stable level, the mechanics also returned to normal.

Angiogenesis in tumors is necessary if the tumor is to grow to any substantial mass beyond that which can be supplied by simple diffusion from the existing circulation. In the lung, these new blood vessels to primary tumors normally arise from the bronchial circulation, ${ }^{7,8}$ which has a prolific capacity for angiogenesis. In the process of this growth, new arterial anastomoses often form between the bronchial and pulmonary circulations. These anastomoses allow the pulmonary circulation to supply nutrient flow to the tumor even if the systemic sources are embolized. ${ }^{8}$ The vascular supply for metastatic lung tumors is more variable, and there is good radiographic evidence for angiogenesis in the pulmonary circulation that supplies these tumors. ${ }^{9}$ True angiogenesis in pulmonary blood vessels of developed lungs is rare, and the process that occurs in these secondary tumors may be similar to what occurs after pneumonectomy in mature animals, when one lung enlarges and grows into the space previously occupied by the excised lung. ${ }^{13}$

In addition to supplying blood flow to tumors, angiogenesis occurs in all tissues when the systemic blood supply is compromised. ${ }^{14}$ Recent studies have followed the time course of angiogenesis after ligation of the femoral artery in the mouse. ${ }^{15}$ Blood flow recovery in the ischemic hindlimb showed a time course very similar to what we observed in the lung, with little change occurring in the first week and then reaching a plateau by 21 days. In systemic tissues the ischemia will always lead to both hypoxia and impaired metabolism. The key role of oxygen level in some situations is demonstrated by experimental work on the development of the retinal vasculature. ${ }^{16,17}$ Generation of new blood vessels in the retina is directly correlated with the level of hypoxia and can be prevented with excess oxygen. ${ }^{16}$ In the lung, however, ischemia caused by hypoxia is clearly not an issue, because the lung is actually better oxygenated when there is no perfusion by deoxygenated venous blood. To the extent that this systemic angiogenesis to the lung has similarities to that in adult systemic tissues after circulatory obstruction, we suggest that hypoxia is not an important or even an essential trigger.

Although there are a host of growth factors, hormones, and chemokines that regulate angiogenesis, ${ }^{4,5}$ release of angiopoietin-1 by mesenchymal cells and binding to endothelial cell TIE2 receptors have been implicated in models of angiogenesis. ${ }^{18}$ If this situation obtained in the lung, however, the angiopoietin-1 signal would have to somehow be transmitted through the pleural fluid to the parietal pleural membrane. Although it may seem somewhat unusual for blood vessels to cross the pleural space, there are several instances of this observed in humans and animals. Weibel used a very similar experimental preparation in rats that also used ligation of the left PA. ${ }^{19}$ Because the rat has a bronchial circulation that supplies intraparenchymal airways, he did show a substantial expansion of the bronchial circulation to the lung after ligation. However, in some animals he showed, as in our results, a dense vascularization between the dorsal lung and chest wall with tortuous intercostal arteries. In humans, clinical treatment of hemoptysis often involves embolization of the bronchial arteries. The body can compensate for this vascular obstruction with angiogenesis of new systemic vessels. This new vascularization can arise from many different locations, ${ }^{20}$ and one of these is from intercostal arteries that penetrate the pleural space in a manner very similar to what we have found in mice.

In our experimental model in mice, there was new vascularization to the lung from the region of the chest wall adjacent to the surgical incision in the third intercostal space through which the left pulmonary artery was ligated. Thus surgical wound healing in the thoracic wall seems to play an important role in supplying new blood vessels to the lung through the pleural space. This was confirmed in two additional mice, in which a second surgical incision was made through the seventh left intercostal space after the left pulmonary artery was ligated through the normal incision in the third space. In both of these mice, we observed new vascular plexuses adjacent to both of the thoracic incisions. Surgical trauma per se, however, is not sufficient to generate systemic blood flow to the mouse lung. Sham-operated mice, with no pulmonary artery ligation, do not even demonstrate adhesions to the chest wall, let alone new blood vessels to the lung. In another pair of mice, we also attempted to determine whether pulmonary vascular ischemia in close proximity to the site of thoracotomy was necessary for angiogenesis. That is, might there be an angiogenic factor released by the ischemic lung that is distributed throughout the body. In these mice, a second surgical 
incision was made in the third right intercostal space, after the left pulmonary artery was ligated through the normal incision in the third left intercostal space. No adhesions or vascularization to the right lung was observed, indicating that the signal to the thoracic wall occurs by direct contact. One interesting experimental intervention would be to tie the left pulmonary artery through a right-sided thoracotomy. Unfortunately, in the mouse this is not a simple procedure, and several mice failed to survive after our attempts at this surgery. Although we always observed vascularization from the chest wall, as noted earlier we occasionally observed new systemic vascularization passing through the thymus. Thus at the present time it is not clear whether surgical trauma in this mouse model is essential for the angiogenesis to successfully generate a new functional systemic vasculature to the lung. Surgical trauma in the thoracic wall is not required for systemic angiogenesis in other mammals.

One question that arises from our work relates to the observation that an increase in microspheres delivered through systemic arteries was not seen for at least 5 days. How can the lung survive for so many days if there is neither pulmonary nor systemic blood flow? There are several possible explanations for this. The first is that there might be sufficient retrograde flow from pulsations in the left atrium. Flow probes placed on the pulmonary veins of larger animals have clearly shown retrograde flow. ${ }^{21}$ Another possibility is the existence of some systemic flow that we fail to measure with the microsphere method. This could happen for three reasons. If the new vessels located at the pleural surface are smaller than 15 $\mu \mathrm{m}$ in diameter, the microspheres might not be able to enter the lung, despite some plasma and red cells being able to trickle through. In addition, the sensitivity of our method of microsphere analysis is only accurate to $\sim 200$ spheres. So with the 100,000 we injected, we can only reliably measure flows as small as $0.2 \%$ of cardiac output. Because we did observe vessels in the lung filled with normal looking red blood cells, either these vessels were communicating with the left atrium, or there was some systemic circulation (perhaps through fibrous attachments) that was too small for us to quantify. Figure 6B clearly shows a filled blood vessel on the pleural surface that is larger than $15 \mu \mathrm{m}$, but because so little is known about the source of the blood vessels on the normal mouse lung visceral pleura, we cannot distinguish between these possibilities. It is worth noting, however, that there were several animals in which there did appear to be some measurable systemic blood flow to the right lung (Figure 1), so perhaps there is a very small amount of perfusion sufficient to maintain tissue viability for days. In contrast to an explanation invoking the lack of penetration of spheres, there is the other extreme, that there are early anastomotic connections that are actually too large to trap and hold even 15- $\mu \mathrm{m}$ spheres. Experimental PA occlusions in larger species have demonstrated the presence of greatly enlarged septal capillaries. ${ }^{2}$ If this had occurred in the mice, then our measurements might be underestimating the actual flow. At the present time, we cannot choose from among these possible explanations. Perhaps further studies using smaller and larger microspheres will provide further insight.

Related to this issue is the nature of the anatomical connections in the lung to the original pulmonary vasculature. Do the new systemic vessels connect to the old pulmonary arteries as the bronchial blood vessels do in other species, ${ }^{2,19}$ or are the connections at the capillary or pulmonary venous level? We have not performed a systematic histological study that would enable us to address this question definitively. However, it would make teleological sense for the new circulation to link up in some manner to the original pulmonary vasculature, as has been described for the circulation of primary lung tumors. $^{7,22}$ Flow from the aorta would clearly have minimal influence on gas exchange, but it would maintain the viability of the pulmonary tissues.

One final note relates to the basic anatomy of the mouse lung. Our experiments with microspheres injected into the left heart confirm the absence of a functional bronchial circulation to the parenchymal airways of the normal mouse. Although we only report a systematic study here in the C57BL/6 mice, we have injected microspheres into several other mouse strains. None of the strains we have examined have a systemic circulation supplying the airways in the parenchyma that can be accurately quantified with microspheres (ie, less than $0.2-0.3 \%$ of cardiac output). This finding is in agreement with the observations of Verloop, ${ }^{6}$ who studied the systemic blood vessels to the lung by using casting material. He was able to show a few bronchial blood vessels at the level of the mainstem bronchi, but he found little evidence for this systemic circulation after the airways entered the parenchyma. The reason for this is likely based on the very small wall thickness of mouse airways. A normal mouse intraparenchymal airway is composed of epithelial cells and a thin smooth muscle layer. Outside this smooth muscle, there is a layer of alveolar capillaries and larger vessels where alveolar walls attach. Apparently these pulmonary blood vessels are sufficient to supply nutrients to the epithelium and smooth muscle without the need for a separate systemic airway circulation. The fact that there is no normal systemic circulation raises questions regarding interpretations of numerous experimental studies using murine models of airways disease, where inflammatory cells lavaged from the lungs are thought to be involved in airway responsiveness. ${ }^{23-25}$ In the mouse, inflammatory cell adhesion and migration into the intraparenchymal airways must occur through capillaries of the pulmonary circulation. This is in sharp contrast to the transit through capillaries of the systemic bronchial circulation that occurs in humans and all other mammals.

In summary, we suggest that this model may offer insights into the process of angiogenesis in general and, more specifically, in the lung. The mouse has a very different response to pulmonary artery ligation compared to all larger mammals. Whether the growth factors and stimuli inducing new vessel growth are unique in mice or the large airway vessels are resistant to these factors remain to be determined. The mouse thus may provide a 
unique model of lung angiogenesis that may have important implications for general pathophysiology and, more specifically, for the growth and potential resolution of lung tumors.

\section{References}

1. Charan NB, Carvalho P: Angiogenesis in bronchial circulatory system after unilateral pulmonary artery obstruction. J Appl Physiol 1997, 82:284-291

2. Schlaepfer K: Ligation of the pulmonary artery of one lung with and without resection of the phrenic nerve. Arch Surg 1924, 9:25-94

3. Michel RP, Hakim TS, Petsikas D: Segment vascular resistance in postobstructive pulmonary vasculopathy. J Appl Physiol 1990, 69: 1022-1032

4. Risau W: Mechanisms of angiogenesis. Nature 1997, 386:671-674

5. Hanahan D: Signaling vascular morphogenesis and maintenance. Science 1997, 277:48-50

6. Verloop MC: On the arteriae bronchiales and their anastomosing with the arteria pulmonalis in some rodents; a micro-anatomical study. Acta Anat 1949, 7:1-32

7. Jonas AM, Carrington CB: Vascular patterns in primary and secondary pulmonary tumors in the dog. Am J Pathol 1969, 56:79-95

8. Muller K, Meyer-Schwickerath M: Bronchial arteries in various stages of bronchogenic carcinoma. Path Res Pract 1978, 163:34-46

9. Milne EN, Zerhouni EA: Blood supply of pulmonary metastases. J Thorac Imaging 1987, 2:15-23

10. Darke CS, Lewtas NA: Selective bronchial arteriography in the demonstration of abnormal systemic circulation in the lung. Clin Radiol 1968, 19:357-367

11. Ewart SL, Levitt RC, Mitzner W: Respiratory system mechanics in mice measured by end-inflation occlusion. J Appl Physiol 1995, 79: 560-566

12. Goldberg HS, Mitzner W, Adams K, Menkes H, Lichtenstein S, Permutt S: Effect of intrathoracic pressure on pressure-volume characteristics of the lung in man. J Appl Physiol 1975, 38:411-417
13. Rudolf AM, Neuhauser EB, Golinko RJ, Auld PA: Effects of pneumonectomy on pulmonary circulation in adult and young animals. Circ Res 1961, 9:856-861

14. Folkman J: Clinical applications of research on angiogenesis. N Engl J Med 1995, 333:1757-1763

15. Couffinhal T, Silver M, Zheng LP, Kearney M, Witzenbichler B, Isner JM: Mouse model of angiogenesis. Am J Pathol 1998, 152:1667-1679

16. Alon T, Hemo I, Itin A, Pe'er J, Stone J, Keshet E: Vascular endothelial growth factor acts as a survival factor for newly formed retinal vessels and has implications for retinopathy of prematurity. Nat Med 1995, 1:1024-1028

17. Stone J, Itin A, Alon T: Development of retinal vasculature is mediated by hypoxia-induced vascular endothelial growth factor (VEGF) expression by neuroglia. J Neurosci 1995, 15:4738-4747

18. Folkman J, D'Amore PA: Blood vessel formation: what is its molecular basis? Cell 1991, 87:1153-1155

19. Weibel ER: Early stages in the development of collateral circulation to the lung in the rat. Circ Res 1960, 8:353-376

20. Keller FS, Rosch J, Loflin TG, Nath PH, McElvein RB: Nonbronchial systemic collateral arteries: significance in percutaneous embolotherapy for hemoptysis. Radiology 1997, 164:687-692

21. Morgan BC, Dillard DH, Guntheroth WG: Effect of cardiac and respiratory cycle on pulmonary vein flow, pressure, and diameter. J Appl Physiol 1966, 21:1276-1280

22. Cudkowicz L: The Human Bronchial Circulation in Health and Disease. Baltimore, Williams and Wilkins, 1968, pp 251-269

23. Wills-Karp M, Luyimbazi J, Xueying X, Schofield B, Neben TY, Karp CL, Donaldson D: Interleukin-13: Central mediator of allergic asthma. Science 1998, 282:2258-2261

24. Longphre M, Zhang L, Harkema JR, Kleeberger SR: Ozone-induced pulmonary inflammation and epithelial proliferation are partially mediated by PAF. J Appl Physiol 1999, 86:341-349

25. De Sanctis GT, MacLean JA, Qin S, Wolyniec WW, Grasemann H, Yandava CN, Jiao A, Noonan T, Stein-Streilein J, Green FH, Drazen JM: Interleukin-8 receptor modulates IgE production and B-cell expansion and trafficking in allergen-induced pulmonary inflammation. J Clin Invest 1999, 103:507-515 